\title{
The diagnostic conundrum of Florid Reactive Periostitis: A case report and review of the literature
}

\author{
Ashton H. Goldman, Jessica A. Frankenhoff * \\ Department of Orthopaedic Surgery, School of Medicine, Virginia Commonwealth University, Richmond, Virginia, United States
}

Received: December 14, 2015

Accepted: January 14, 2016

Online Published: January 25, 2016

DOI: $10.5430 /$ css.v2n2p1

URL: http://dx.doi.org/10.5430/css.v2n2p1

\begin{abstract}
We report on a case of digital benign fibroblastic proliferative lesion that aggressively recurred after multiple excisions and showed histologic progression from a Fibrosseous Pseudotumor (FOPT) to Florid Reactive Periostitis (FRP). We recommend caution on incisional biopsies that do not excise the lesion fully based on the clinical course, radiographic, and histologic findings. Our case demonstrates the protean nature of this entity and the difficulty in obtaining an accurate diagnosis based on the clinical course and radiographic and histologic findings.
\end{abstract}

Key Words: Recurrence, Fibular bone graft, Ossificans, Fasciitis, Pseudotumor, Hand, Reactive

\section{CASE REPORT}

A 6-year-old right-hand dominant African-American boy presented to an outside hospital with an enlarging, non-painful mass at the mid-volar-ulnar aspect of his right long finger's proximal phalanx. Plain films noted a "subtle indentation of the ulnar cortex of the proximal phalanx". During attempted excision, the mass was found "matted to the tissue" with "no capsular margin", and an incisional biopsy was performed. Final pathology concluded it was a benign myofibroma.

Two months later, the mass appeared enlarged. Repeat $x$-rays demonstrated periosteal calcification without cortical erosion. An MRI showed the tumor wrapping around the bone under the extensor mechanism and volar to the flexor tendons with "intense bone marrow edema throughout the proximal phalanx sparing the epiphyses". A soft tissue excision was performed, sacrificing the ulnar digital artery, the volar half of the collateral ligament and the ulnar portion of the volar plate. Multiple pathologists interpreted this specimen as a Fibrosseous Pseudotumor (FOPT) of the digit with the conclusion that the original biopsy must have been a peripheral sampling early in its evolution.

Five months after excision, he presented to our hospital for the first time having developed a larger, now painful, recurrence. MRI revealed a mass that had dense peripheral calcifications and an heterogeneous central area (see Figure 1). The radiologist suspected dedifferentiation into a more aggressive process with potential malignant degeneration. He was taken for biopsy with the goal of obtaining both osseous and soft tissue components. Pathology revealed zoning: bony maturation with trabeculae at the periphery and immature osteoid in the central portion, without evidence of malignant atypia or nuclear pleomorphism. These finding were now more consistent with Florid Reactive Periostitis (FRP). His mother was given the choice of ray amputation or en-bloc surgical excision.

The family elected to proceed with an excision of the lesion

\footnotetext{
* Correspondence: Jessica A. Frankenhoff; Email: jessica.frankenhoff@vcuhealth.org; Address: Department of Orthopaedic Surgery, School of Medicine, Virginia Commonwealth University, Richmond, Virginia, P.O. Box 980153, United States.
} 
along with the central $80 \%$ of the proximal phalanx diaphysis followed by interpositional fibular bone grafting. All soft tissue including periosteum associated with the mass was attempted to be excised grossly. Pathology showed nodular fasciitis-like stromal proliferation, ongoing osseous maturation and impingement of the mass onto the cortex of the proximal phalanx (see Figures 2-4).

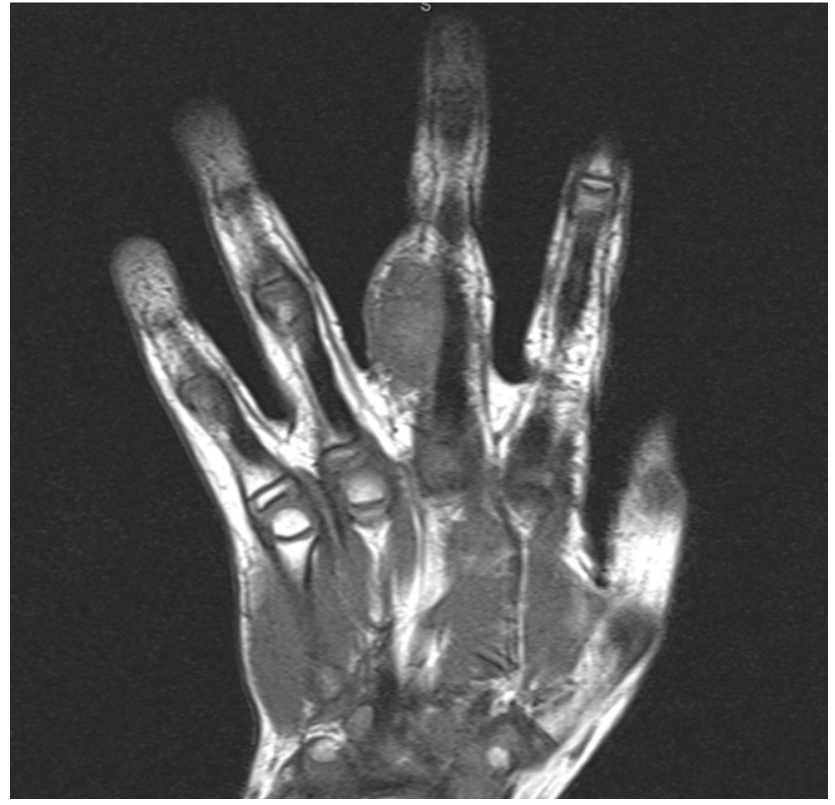

Figure 1. Enlarging recurrent mass over long finger proximal phalanx

The fibular bone graft healed well with good incorporation and function (see Figure 5). The fibular donor site healed with new bone without issue (see Figure 6). Four months after surgery, the mass recurred for a third time along the central aspect of the fibular graft (see Figure 7), and it was decided to proceed with ray resection. Final pathology was consistent with FRP. At the six-month follow-up, he had healed wounds, good function, and no recurrence.

\section{Discussion}

The differential diagnosis for benign fibroblastic proliferative lesions about the hand is difficult owing to the fact that multiple names have been used throughout the literature. Our case is the first to demonstrate histologic progression over multiple biopsies from a myofibroma, FOPT and finally FRP. The difficult diagnosis and potential temporal relationships of these related lesions are not well defined. Due to their rarity, our understanding is limited to case series and case reports. The largest series, found in the pathology literature, demonstrates a spectrum of disease with a close resemblance to a subcutaneous variant of myositis ossificans. ${ }^{[1,2]}$

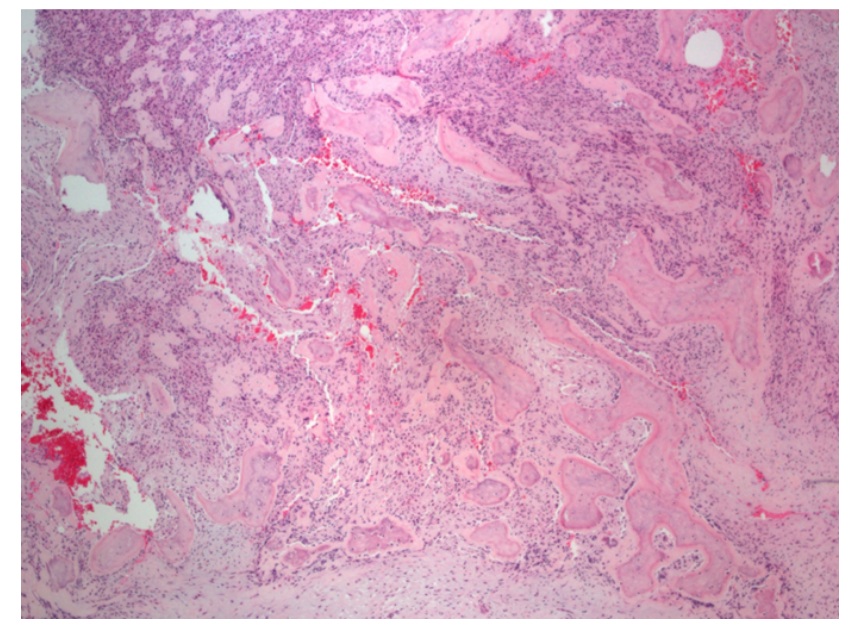

Figure 2. Low power histology demonstrating bony trabeculae with nodular fasciitis like stroma without evidence of cellular atypia

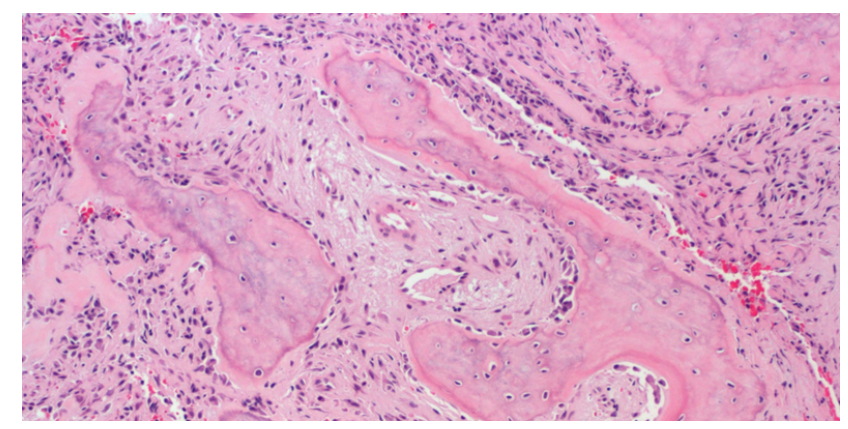

Figure 3. Medium power histology demonstrating bony trabeculae with nodular fasciitis like stroma without evidence of cellular atypia

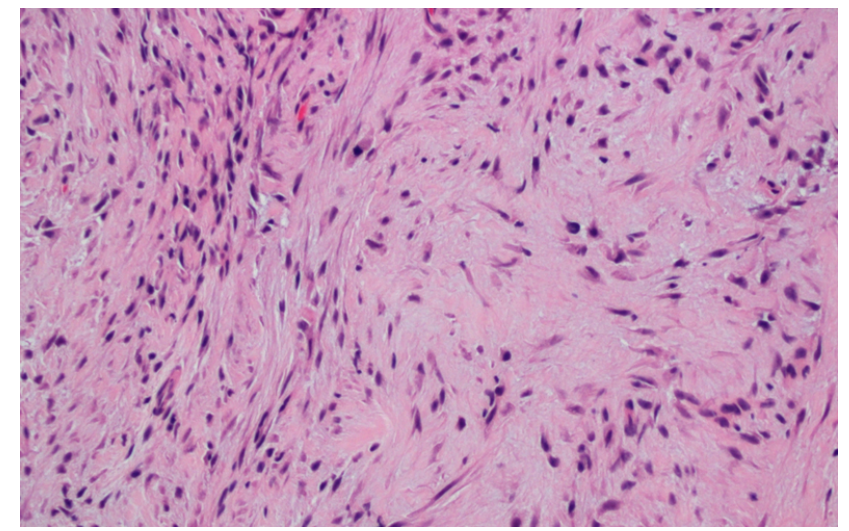

Figure 4. High power histology demonstrating bony trabeculae with nodular fasciitis like stroma without evidence of cellular atypia

Clinically patients present with a painful, fusiform, swollen mass, typically on the index or ring finger that is sometimes associated with prior trauma or repetitive manual activity. ${ }^{[1-3]}$ Over $90 \%$ can be found at the proximal or middle 
phalanx. ${ }^{[2,3]}$ Based off alarming clinical findings, one must consider osteomyelitis, osteogenic sarcoma, and other malignancies. ${ }^{[4-6]}$

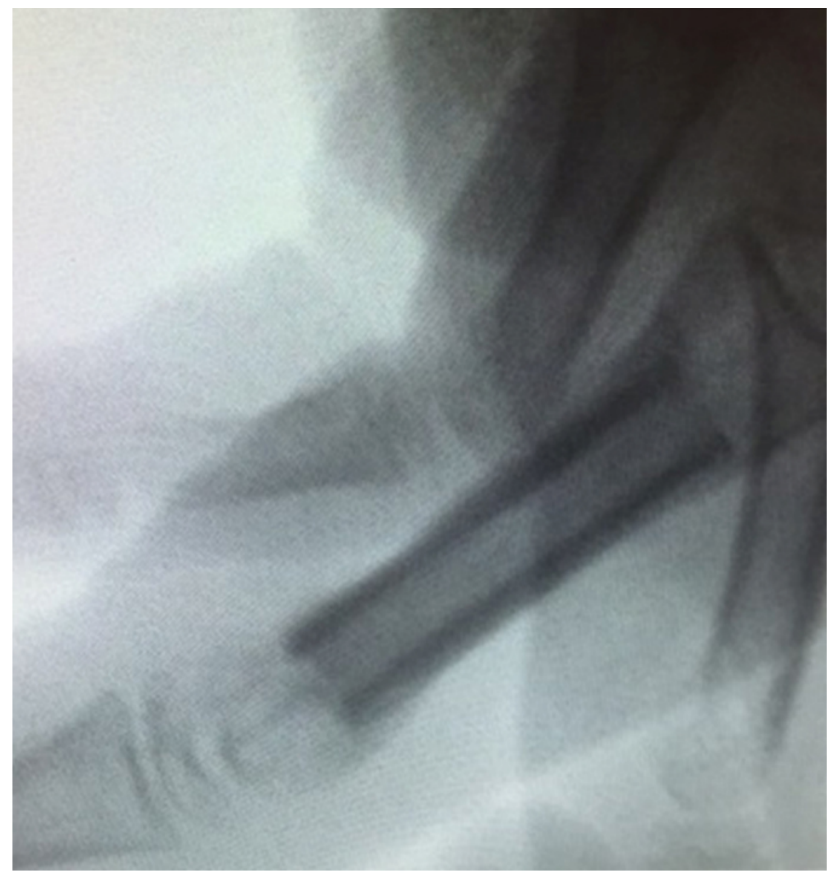

Figure 5. Ongoing healing of fibular strut graft

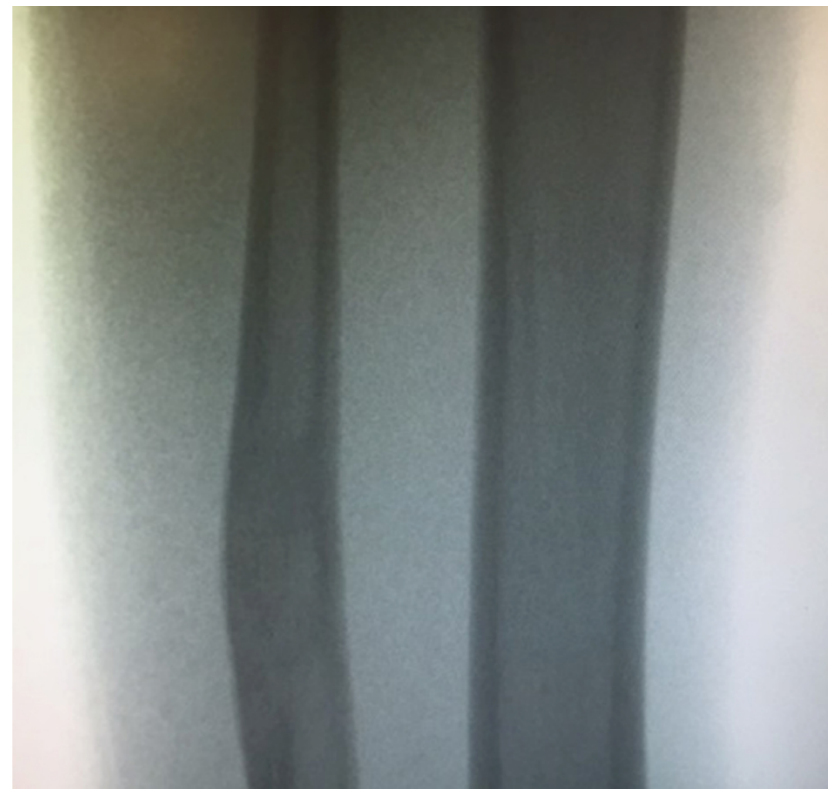

Figure 6. Healed fibular strut graft donor site

Unfortunately plain radiographs demonstrating periosteal reaction, cortical erosions, and calcifications do not always aid in the diagnostic challenge as these features vary. ${ }^{[1-3,7]}$ Spjut and Dorfman noted an agressive periosteal reaction noted on radiographs thereby naming it FRP. ${ }^{[3]}$ Likewise Dupree Published by Sciedu Press and Enzinger found less than $50 \%$ of their 21 cases involving the periosteum concluding that the periosteum is simply a reactive bystander, providing the name fibro-osseous pseudotumor. $^{[7]}$ Other names, including nodular fasciitis ${ }^{[8]}$ and parosteal fasciitis ${ }^{[9]}$ have been commonly used to describe the same entity. MRI findings can be consistent with parosteal osteosarcoma, thereby further confusing the clinical picture. ${ }^{[4]}$ Our patient demonstrated potential malignant degeneration based on MRI leading to further confusion.

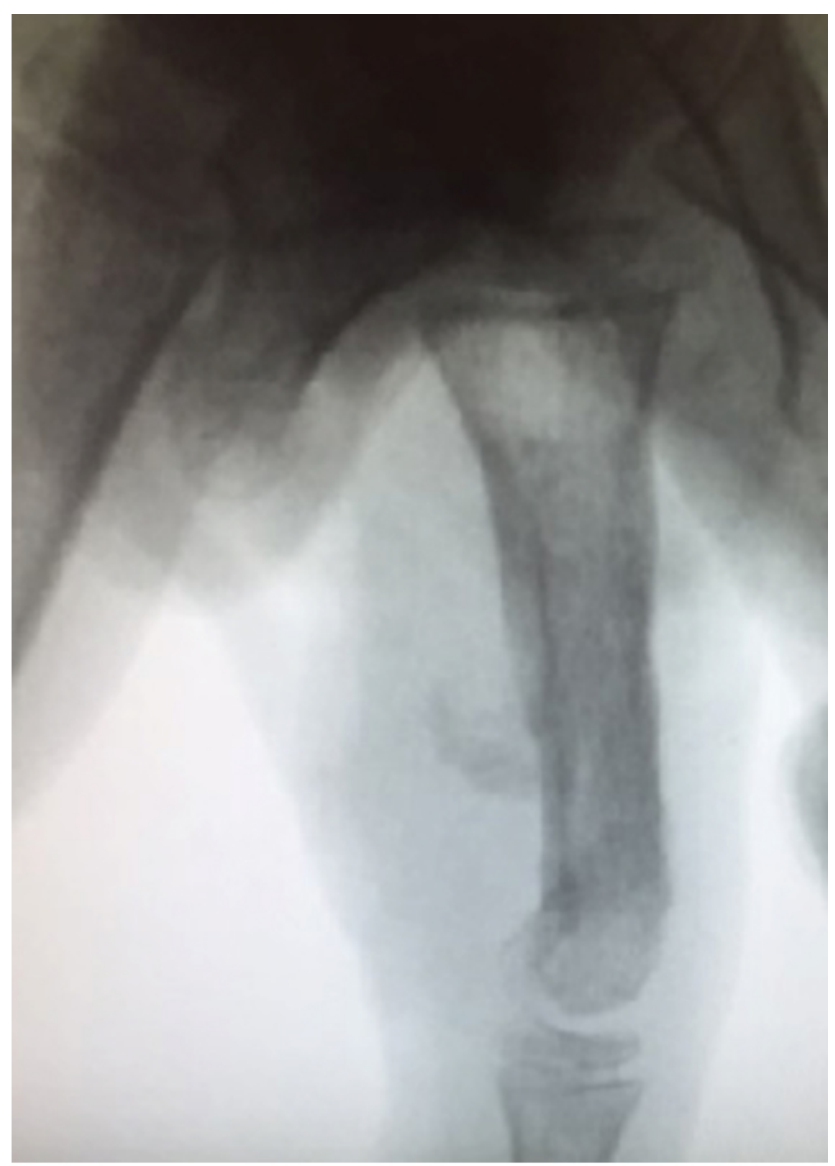

Figure 7. Recurrence of mass over proximal phalanx of the long finger at the central aspect of the strut graft

In the only prospective study to date, Sundaram et al. followed three patients ages 11 to 34 with a presumptive radiographic diagnosis of FRP. They watched the lesions radiographically rather than biopsy them based on epidemiological studies showing that while FRP is rare, osteogenic sarcoma is even more so in the fingers and it rarely presents under age 40. They found that the masses showed a stable increase in size and periosteal reaction. ${ }^{[10]}$ Similar to our case, the periosteal reaction developed later, possibly demonstrating the aforementioned temporal relationship of FOPT and FRP. Based off their observations, they believed these lesions were related to myositis ossificans and heterotopic ossification, 
where operating too early could lead to recurrence. ${ }^{[10]}$

The overlapping histological relationship between FRP, FOPT, and Myositis Ossificans (MO) is even more ambiguous. The zoning phenomenon with mature bone cells at the periphery and immature osteoid at the central portion is representative of myositis ossificans, but also is seen in over $50 \%$ of patients with FRP..$^{[2,5,7,11]}$ However, it is typically more developed with MO, and thus the zoning with FRP is referred to as incomplete. ${ }^{[5,12,13]}$ Fasciitis-like stroma with fibroblast, osteoblast, osteoclast, moderate cellularity, no atypia and intramembranous woven bone production can be seen with both FOPT and FRP, but unlike FOPT, the ossification center of FRP is attached to bone. ${ }^{[2,14]}$ Histochemically, MO, FOPT, and FRP stain positive for muscular vimentin, actin and the proliferative $\mathrm{Ki}-67$ marker while staining negative for MAK-6, S-100 and desmin. ${ }^{[2,13,15]}$

Even once a presumptive diagnosis is made based on clinical, radiographic and histologic findings, the natural history can be unpredictable as four documented possibilities have been reported. It can spontaneously resolve; be cured after excision, recur in an insidious pattern, or recur more aggressively with surmised aggravation from previous surgical trauma, as in our case. Many have had great success with excision, ${ }^{[3,6,7,10,15-17]}$ and while there are no reports of FRP being treated medically, spontaneous resolution has been documented. Misdiagnosis has occurred, which has led to unnecessary early radical amputations. ${ }^{[8]}$

Many authors contend that FRP is a subcutaneous variant on a continuum with $\mathrm{MO},{ }^{[1,2,6,7,13]}$ but this is not universally accepted. ${ }^{[11,16]}$ Nonoperative non-steroidals or radiation have therefore been suggested as potential treatments. ${ }^{[10,18]}$ Along those lines, rapid recurrence following surgical excision or inadequate resection before the lesion matures has been implicated. ${ }^{[10,15,19]}$ Other authors indicate the inciting surgical trauma itself can spur a more aggressive recurrence, ${ }^{[11,18]}$ and this is a potential reason demonstrating the histological progression of our lesion from FOPT to FRP. To date, malignant transformations have not been encountered. ${ }^{[1,14,18]}$
Our case demonstrated varying radiographic features including progressive periosteal reaction and recurrence of the lesion abutting the fibular strut graft. Histologically, the diagnosis progressed from myofibroma, FOPT and finally FRP. While observation was the chosen course following the first diagnosis, the treatment plan was revised with the lesion's aggressive transformation. Following the diagnosis of FRP, we were hopeful that resecting the matted periosteum along with the bone and mass would confer a cure. Unfortunately, this did not prove to be the case. With the bone fully excised, we surmise that either the origin of the lesion was in the soft tissue, or that we did not, in fact, excise some microscopic periosteal components in our resection. Whether the outcome would have been different had en-bloc excision been the first surgery versus the fourth is unclear. The fibular graft was chosen as a less morbid option over an iliac graft in order to preserve the growth plate and limit morbidity due to its regrowth potential. Following the fibular strut grafting, a trial of non-steroidals or radiation may have aided in preventing recurrence, but this was not done.

Fibrous masses of unknown origin in the hands will likely continue to challenge practitioners, as they are exceedingly rare. We agree with other authors that FRP has significant similarities to FOPT and MO. We highlight a potential histologically proven temporal relationship between myofibroma, FOPT and FRP. We suggest caution in performing incisional biopsies as surgical trauma can precipitate a more aggressive lesion. Following excision, a trial of non-steroidals or radiation may be considered based on a potential relationship to other aberrant osseous growths. Future, larger studies are needed to better characterize these lesions, which in turn could aid in a focused treatment strategy, potentially avoiding ray resection.

\section{CONFlicts of INTEREST Disclosure}

The authors declare that they have no conflict of interest. This article does not contain any studies with human or animal subjects. Informed consent was obtained from the participant included in the case report.

\section{REFERENCES}

[1] De Silva M, Reid R. Myositis ossificans and fibroosseous pseudotumor of digits: a clinicopathological review of 64 cases with emphasis on diagnostic pitfalls. Int J. of Surg Path. 2003; 11: 187-195. http://dx.doi.org/10.1177/106689690301100305

[2] Moosavi C, Al-Nahar L, Murphey M, et al. Fibrosseous pseudotumor of the digit: a clinicopathologic study of 43 new cases. Ann Diag Path. 2008; 12: 21-28. PMid: 18164411. http://dx.doi.org/10. 1016/j.anndiagpath.2007.02.001
[3] Spjut H, Dorfman H. Florid reactive periostitis of the tubular bones of the hands and feet: a benign lesion which may simulate osteosarcoma. Am J Surg Path. 1981; 5: 423-434. http://dx.doi .org/10.1097 /00000478-198107000-00002

[4] Gao Z, Wang J, Wang Z, et al. Florid Reactive Periostitis of the Metacarpal and Phalanx: 2 Case Reports. J. Hand Surg. 2013; 38: 2134-2137. PMid: 24206975. http://dx.doi.org/10.1016/j .jhsa.2013.08.115

[5] Jambhekar N, Desai S, Puri A, et al. Florid reactive periostitis 
of the hands. Skel Rad. 2004; 33: 663-665. PMid: 15248034. http://dx.doi.org/10.1007/s00256-004-0760-3

[6] Coleman R. Fibro-osseous pseudotumour of the digit-amputation for a benign but aggressive lesion. J Hand Surg (Br and Eur Vol). 2005; 30: 504-506. PMid: 15992973. http://dx.doi.org/10.1016/j .jhsb.2005.05.005

[7] Dupree W, Enzinger F. Fibro-osseous pseudotumor of the digits. Cancer. 1986; 58: 2103-2109. http: //dx.doi.org/10.1002/1097-0142(19861101)58: 9<2103: : AID-CNCR2820580923>3.0. C0;2-C

[8] McCarthy E, Ireland D, Sprague B, et al. Parosteal (nodular) fasciitis of the hand. A case report. J Bone \& Joint Surg. 1976; 58: 714-716.

[9] Hutter R, Foote Jr F, Francis K, et al. Parosteal fasciitis: a selflimited benign process that simulates a malignant neoplasm. Am J Surg. 1962; 104: 800-807. http://dx.doi .org/10.1016/000 2-9610 (62) 90448-8

[10] Sundaram M, Rotman M, Howard R, et al. Florid reactive periostitis and bizarre parosteal osteochondromatous proliferation: pre-biopsy imaging evolution, treatment and outcome. Skel Rad. 2001; 30: 192 198. http://dx.doi.org/10.1007/s002560100343

[11] Patel M, Desai S. Pseudomalignant osseous tumor of soft tissue: a case report and review of the literature. J Hand Surg. 1986; 11: 66-70. http://dx.doi.org/10.1016/S0363-5023(86)80105-8

[12] Nalbantoglu U, Gereli A, Kocaoglu B, et al. Fibro-osseous pseudotumor of the digits: a rare tumor in an unusual location. J Hand Surg. 2008; 33: 273-276. PMid: 18294555. http://dx.doi.org/10.10 $16 / j \cdot j h s a .2007 .10 .005$
[13] Sleater J, Mullins D, Chun K, et al. Fibro-osseous pseudotumor of the digit: a comparison to myositis ossificans by light microscopy and immunohistochemical methods. J Cutaneous Path. 1996; 23: 373-377. http://dx.doi.org/10.1111/j.1600-056 $0.1996 . t b 01313 . x$

[14] Craver R, Correa-Gracian H, Heinrich S. Florid reactive periostitis. Hu Path. 1997; 28: 745-747.

[15] Coleman R. Fibro-osseous pseudotumour of the digit-amputation for a benign but aggressive lesion. J Hand Surg (Br and Eur Vol). 2005; 30: 504-506. PMid: 15992973. http://dx.doi.org/10.1016/j .jhsb.2005.05.005

[16] Kovach J, Truong L, Kearns R, et al. Florid reactive periostitis. J Hand Surg. 1986; 11: 902-905. http://dx.doi.org/10.1016/S 0363-5023(86) 80248-9

[17] Schütte H, Van der Heul R. Pseudomalignant, nonneoplastic osseous soft-tissue tumors of the hand and foot. Radiology. 1990; 176: 149. 153. PMid: 2112767. http://dx.doi.org/10.1148/radiology .176 .1 .2112767

[18] Rogers G, Brzezienski M. Florid reactive periostitis of the middle phalanx: a case report and review of the literature. J Hand Surg. 1999; 24: 1014-1018. PMid: 10509281. http://dx.doi.org/10.1053 /jhsu.1999.1014

[19] Yuen M, Friedman L, Orr W, et al. Proliferative periosteal processes of phalanges: a unitary hypothesis. Skel Rad. 1992; 21: 301-303. http://dx.doi.org/10.1007/bf00241768 